\title{
Single Versus Multi-Specialty Operative Teams: Association with Perioperative Mortality after Endovascular Abdominal Aortic Aneurysm Repair
}

\author{
LAURA M. MAZER, M.D., M.S. ${ }^{*}$ ELLIOT L. CHIAKOF, M.D., Ph.D. ${ }^{*}$ PHILIP P. GOODNEY, \\ M.D., M.S. ${ }^{\dagger}$, MATTHEW S. EDWARDS, M.D., M.S. ${ }^{\ddagger}$, and MATTHEW A. CORRIERE, M.D., \\ M.S.§ \\ "Department of Surgery, Beth Israel Deaconess Medical Center, Boston, Massachusetts \\ tSection of Vascular Surgery, Dartmouth-Hitchcock Medical Center, Lebanon, New Hampshire \\ ‡Department of Vascular Surgery, Wake Forest Medical Center, Winston-Salem, North Carolina \\ §Surgical Service, Atlanta VA Medical Center, Division of Vascular Surgery and Endovascular \\ Therapy, Emory University School of Medicine, Atlanta, Georgia
}

\section{Abstract}

Endovascular abdominal aortic aneurysm repair (EVAR) requires both endovascular and open surgical skills. Although usually performed by a single operating specialist, EVAR may alternatively involve multiple teams from different specialties performing separate procedural components. We examined the relative frequencies of single versus multi-specialty EVAR in the 2005 to 2008 American College of Surgeons National Surgical Quality Improvement Participant Use Datafile and explored the influence of multi-specialty EVAR on 30-day mortality. EVARs were identified and classified as single or multiple-specialty procedures based on Current Procedural Terminology codes. Baseline and procedural characteristics were compared using $\chi^{2}$ or Fisher's exact test for categorical variables and $t$ test for continuous variables. The association between multi-specialty EVAR and 30-day mortality was examined using a multivariate logistic regression model. Of 7269 EVAR patients identified, 7086 were single and 183 were multispecialty. Multi-specialty patients had higher frequency of brachial or iliac artery exposure and longer operative times, but were otherwise similar in baseline and procedural characteristics. In the multivariate model, multi-specialty EVAR was associated with increased risk of 30-day mortality (odds ratio 2.35; 95\% confidence interval 1.08-5.11; $P$ value 0.031 ). Multi-specialty participation in EVAR procedures is associated with significantly higher 30-day mortality. Further research is warranted to determine whether multi-specialty participation reflects provider experience, institutional protocols, procedural complexity, non-surgical or other factors.

Endovascular aortic aneurysm repair (EVAR) is presently the most common method for treatment of infrarenal abdominal aortic aneurysms (AAA) in the United States. ${ }^{1}$ Before the initial description of EVAR by Parodi et al., ${ }^{2}$ open surgical repair was the primary method

\footnotetext{
Address correspondence and reprint requests to Matthew A. Corriere, M.D., M.S., Surgical Service, Atlanta VA Medical Center, Department of Vascular Surgery and Endovascular Therapy, Emory University School of Medicine and the Department of Epidemiology, Rollins School of Public Health, Emory University, 101 Woodruff Circle, Suite 5105, Atlanta, GA 30322. Matthew.corriere@emoryhealthcare.org.

Disclaimer

The American College of Surgeons National Surgical Quality Improvement Program and the hospitals participating in the ACS NSQIP are the source of the data used herein; they have not verified and are not responsible for the statistical validity of the data analysis or the conclusions derived by the authors.
} 
for AAA treatment. The subsequent development of commercially available EVAR devices prompted many surgeons to acquire endovascular skills, and also resulted in the addition of AAA treatment to the practice of many specialists with endovascular skills who do not perform open surgical repair.

Although EVAR can be performed using completely percutaneous methods, open arterial exposure is frequently necessary when access vessels are severely diseased or have a significant diameter mismatch with endograft delivery systems. As a result, EVAR is usually performed by a single operator with both open and endovascular skills, but also may be performed by multiple specialists with complementary skills performing separate procedural components. The relative frequency with which EVAR is performed by single versus multi-specialty operators is unknown, and related influences on perioperative outcomes have not been characterized. We analyzed the 2005 to 2008 American College of Surgeons National Surgical Quality Improvement (ACS-NSQIP) Participant Use Data File to determine the frequency of EVAR performance by single versus multi-specialty operative teams and to explore associations with procedural characteristics and 30-day mortality.

\section{Methods}

\section{Study Population}

The ACS-NSQIP collects patient-level preoperative, procedural, and postoperative data including 30-day mortality and morbidity outcomes. Case capture is based on a systematic sampling process based on an 8-day cycle; the program monitors case selection and case mix on a weekly basis to ensure appropriate selection. Data collection occurs in a standardized fashion and is validated by trained Surgical Clinical Reviewers at each site. The accuracy and reproducibility of the ACS-NSQIP data has been previously demonstrated. ${ }^{3,4}$

\section{Patient Selection}

We analyzed the 2005-2008 ACS-NSQIP Participant Use Data File. EVAR procedures were identified using the Current Procedural Terminology (CPT) codes listed (Table 1). All available CPT fields $(n=21)$ were queried, and patients were excluded from analysis if any CPT code or postoperative diagnosis [based on International Classification of Diseases, 9th revision (ICD-9) code] indicated other major operations during the same anesthetic that are not routinely performed in combination with EVAR (examples include thoracic aortic repair, coronary artery bypass, intestinal resection, and splenectomy). Multi-specialty EVARs were identified based on concurrent CPT codes, which within ACS-NSQIP designate additional operative procedures performed during the same anesthesia by a surgical team from a different specialty or service. Multiple operators of the same specialty (for example, two vascular surgeons participating in the same case) do not generate concurrent CPT codes. EVARs were designated as having multi-specialty operative teams when concurrent CPT codes indicated the performance of individual EVAR components (including endograft insertion, open arterial exposure for endograft delivery, and radiologic supervision and interpretation) by multiple teams of different specialties.

\section{Statistical Analysis}

Group-wise comparisons of preoperative risk factors and procedure-related variables based on single- versus multi-specialty operative team status were performed using $\chi^{2}$ test of independence or Fisher's exact test for categorical variables and $t$ tests for continuous variables. Risk of 30-day mortality was evaluated using a multivariate logistic regression analysis; candidate covariates were identified based on a literature review of factors associated with mortality after EVAR and included: patient age (patient age $>90$ set to null 
as these values are not recorded in ACS-NSQIP), ${ }^{5,6}$ gender, ${ }^{6}$ American Society of Anesthesiologists (ASA) physical status classification, ${ }^{5,7}$ preoperative sepsis (systemic inflammatory response syndrome, sepsis, and/or septic shock), ${ }^{8}$ emergency procedure status, ${ }^{8}$ recent weight loss, ${ }^{8}$ preoperative requirement for dialysis, ${ }^{6,9}$ dependent functional status, ${ }^{8}$ history of hypertension, ${ }^{6,8}$ use of iliac or brachial artery exposure for endovascular prosthesis delivery, ${ }^{8}$ preoperative white blood cell (WBC) count $\left(1000 / \mathrm{mL}^{3}\right),{ }^{10}$ and preoperative serum creatinine. ${ }^{8,9}$ These variables, along with multi-specialty operative team status, were considered for the logistic regression model using a stepwise selection approach with $P<0.10$ as the significance criterion for model entry and $P \geq 0.05$ as the criterion for retention in the final multivariate model.

\section{Results}

A total of 7269 EVAR procedures were identified and analyzed. Seven thousand and eightysix EVARs $(97.5 \%)$ had all procedure components performed by a single operative team, whereas 183 EVARs $(2.5 \%)$ had multi-specialty operative teams. Preoperative patient characteristics stratified by single versus multi-specialty operative team status are displayed in Table 2. Groups were similar in terms of age, gender, emergent procedure status, and prevalence of preoperative comorbid conditions. Frequencies of dissection or rupture diagnoses were also similar between groups ( $P=0.602$ and 0.688 , respectively). Patients with multi-specialty operative teams had significantly higher mean preoperative WBC count $\left(8.5 \pm 4.2\right.$ vs $\left.7.7 \pm 2.9 \times 1000 / \mathrm{mL}^{3} ; P=0.014\right)$, but rates of preoperative sepsis were similar between groups $(0.62 \%$ vs $1.64 \%, P=0.114)$. Table 3 displays procedural characteristics categorized by single versus multi-specialty repair status. Compared with single specialty EVARs, multi-specialty EVARs had longer mean operative times (238.2 \pm 91 vs $222.4 \pm 87$ minutes; $P=0.034$ ) and more frequent utilization of iliac or brachial artery exposure for endoprosthesis delivery $(6.7 \%$ vs $1.9 \% ; P<0.001)$. We did not identify significant differences in intra operative transfusion rates, primary surgical team specialty, or rates of intraoperative conversion to open aneurysm repair based on multi-specialty team status.

Overall 30-day mortality was 2.5 per cent. Unadjusted mortality was significantly higher in patients with multi-specialty operative teams (6.1\% vs $2.4 \%$; univariate $P<0.006)$. Multispecialty EVAR was also associated with perioperative mortality in multivariate modeling adjusting for other significant risk factors [odds ratio 2.35, 95\% confidence interval (1.085.11); $P=0.031$ ] (Table 4). Other factors associated with 30-day mortality in multivariate modeling included American Society of Anesthesiologists class, preoperative WBC count, emergent procedure status, loss of $>10$ per cent of body weight in the 6 months before surgery, female gender, dependent functional status, age, and use of iliac or brachial artery exposure for endograft delivery (Table 4).

\section{Discussion}

Since its initial description, EVAR has become the most common method of treatment for infrarenal abdominal aortic aneurysm repair. EVAR is less invasive and associated with lower risk of both perioperative mortality and adverse events when compared with open repair, resulting in an increasing utilization of this treatment method by physicians from a variety of specialty backgrounds. ${ }^{11,12}$ Although perioperative outcomes associated with EVAR have been extensively studied, the majority of these analyses have been generated by same-specialty groups performing all procedural components. ${ }^{10,13}$ To our knowledge, the proportion of EVARs performed by multi-specialty teams and the related associations with perioperative outcomes have not been previously characterized. 
In our analysis of the ACS-NSQIP Participant Use Data File, patients undergoing single versus multi-specialty EVAR procedures had similar preoperative characteristics, but procedures performed by multi-specialty operative teams had longer procedures and more frequent utilization of iliac or brachial artery exposure for endograft delivery. In multivariate models adjusting for other reported predictors of perioperative mortality, we observed a significant association between multi-specialty EVAR and perioperative mortality.

Inclusion of EVAR procedures from a large number of centers with specific identification of CPT codes generated by specialists distinct from that of the primary operating team made evaluation of multi-specialty repair possible within ACS-NSQIP. However, the data set does not provide the indication for multi-specialty involvement and only records the specialty of the primary team, limiting additional inferences that can be made from the observed associations. EVAR involves technical skills used with varying frequency by specialists in cardiology, interventional radiology, vascular medicine, and a range of surgical subspecialties (including general, vascular, and cardio-thoracic surgery). Experience and comfort levels of individual practitioners therefore may influence the likelihood of multispecialty participation in a variety of ways.

Multi-specialty participation may occur due to a routine collaborative approach toward EVAR, or instead as the result of unplanned intraoperative consultations generated by technical problems on the part of the primary operator. Collaboration between different specialists may also occur with greater frequency among practitioners with relatively limited experience; multi-specialty EVAR therefore may represent a confounder for physician technical experience or procedural volume. Alternatively, the longer procedure durations and higher rate of nonfemoral arterial access utilization we observed for multi-specialty EVARs may indicate greater procedural complexity in this group. We are unable to determine in this analysis, however, in which instances procedural complexity resulted from or instead was created by involvement of more than one operative team. Because hospitaland physician-level data are not available within the ACS-NSQIP Participant Use Data File, additional analyses with other data sources will be necessary to make more detailed inferences regarding associations between operative team composition and outcomes associated with EVAR.

Specialty influences on EVAR procedural conduct have been described previously. Operator specialty has been shown to impact indications for repair, choice of preoperative imaging modalities, operative technique, and postoperative management. ${ }^{14}$ Additionally, the cooperation of multiple surgical specialties has been shown to impact outcomes in other common operations. ${ }^{15,16}$ Unlike operations where multiple specialties routinely participate in coordinated comanagement of patient care and procedures, EVAR is most often performed by a single specialist. Ambiguity regarding provider roles, responsibilities, and leadership has been identified as contributing to communication breakdown. ${ }^{17}, 18$ Theoretically, the increased number of personnel (both operators and assistants) participating in multi-specialty EVAR procedures would by definition increase procedural complexity independent of patient-related factors. The requirement for a greater amount of verbal communication increases the odds of misunderstandings and hand-off errors, which also have adverse effects on patient outcomes. ${ }^{19}$ Such errors might explain our observations independent of specialty-specific factors.

Our study has several additional limitations that deserve specific mention. First, the ACSNSQIP is defined by surgeon participants; as such, the dataset may not capture EVAR procedures performed outside the operating room or without surgeon participation. Second, ACS-NSQIP uses a sampling approach to case identification and capture, so the procedures analyzed also cannot be considered complete with regard to EVARs performed in the 
operating room. Our analyses therefore cannot be considered completely representative of all EVAR procedures performed at participating centers, and our findings cannot be generalized to single-specialist EVARs performed by nonsurgeons. Third, because we are only able to identify the specialty of the primary surgical team, we were unable to perform more specific analyses of secondary team participation.

Despite these limitations, our study highlights the potential influence of operative team factors on patient outcomes. As interventional medical specialists increase in number, it is likely that multi-specialty operations involving surgeons and nonsurgeons will become increasingly common. Given these considerations, further investigation of associations between adverse events and procedural factors, including the role of communication and delegation between individuals comprising the operative team, is warranted. Such investigations may identify procedural factors that can be modified for potential improvement of patient outcomes.

\section{References}

1. Ng TT, Mirocha J, Magner D, Gewertz BL. Variations in the utilization of endovascular aneurysm repair reflect population risk factors and disease prevalence. J Vasc Surg. 2010; 51:801-9. 809e1. [PubMed: 20347673]

2. Parodi JC, Palmaz JC, Barone HD. Transfemoral intraluminal graft implantation for abdominal aortic aneurysms. Ann Vasc Surg. 1991; 5:491-9. [PubMed: 1837729]

3. Khuri SF, Henderson WG, Daley J, et al. Successful implementation of the Department of Veterans Affairs' National Surgical Quality Improvement Program in the private sector: the Patient Safety in Surgery study. Ann Surg. 2008; 248:329-36. [PubMed: 18650645]

4. Fink AS, Campbell DA Jr, Mentzer RM Jr, et al. The National Surgical Quality Improvement Program in non-veterans administration hospitals: initial demonstration of feasibility. Ann Surg. 2002; 236:344-53. discussion 353-4. [PubMed: 12192321]

5. de Virgilio C, Tran J, Lewis R, et al. Factors affecting long-term mortality after endovascular repair of abdominal aortic aneurysms. Arch Surg. 2006; 141:905-9. discussion 909-10. [PubMed: 16983034]

6. Giles KA, Schermerhorn ML, O’Malley AJ, et al. Risk prediction for perioperative mortality of endovascular vs open repair of abdominal aortic aneurysms using the Medicare population. J Vasc Surg. 2009; 50:256-62. [PubMed: 19249184]

7. Harris PL, Vallabhaneni SR, Desgranges P, et al. Incidence and risk factors of late rupture, conversion, and death after endo-vascular repair of infrarenal aortic aneurysms: the EUROSTAR experience. European Collaborators on Stent/graft techniques for aortic aneurysm repair. J Vasc Surg. 2000; 32:739-49. [PubMed: 11013038]

8. Abedi NN, Davenport DL, Xenos E, et al. Gender and 30-day outcome in patients undergoing endovascular aneurysm repair (EVAR): an analysis using the ACS NSQIP dataset. J Vasc Surg. 2009; 50:486-91. 491, e1-4. [PubMed: 19628363]

9. van Eps RG, Leurs LJ, Hobo R, et al. Impact of renal dysfunction on operative mortality following endovascular abdominal aortic aneurysm surgery. Br J Surg. 2007; 94:174-8. [PubMed: 17117430]

10. Davenport DL, O'Keeffe SD, Minion DJ, et al. Thirty-day NSQIP database outcomes of open versus endoluminal repair of ruptured abdominal aortic aneurysms. J Vasc Surg. 2010; 51:305-9. e1. [PubMed: 19939609]

11. Greenhalgh RM, Brown LC, Kwong GP, et al. Comparison of endovascular aneurysm repair with open repair in patients with abdominal aortic aneurysm (EVAR trial 1), 30-day operative mortality results: randomised controlled trial. Lancet. 2004; 364:843-8. [PubMed: 15351191]

12. Boyle JR, Thompson MM, Sayers RD, et al. Changes in referral practice, workload, and operative mortality after establishment of an endovascular abdominal aortic aneurysm program. J Endovasc Surg. 1998; 5:201-5. [PubMed: 9761570] 
13. Prinssen M, Verhoeven EL, Buth J, et al. A randomized trial comparing conventional and endovascular repair of abdominal aortic aneurysms. N Engl J Med. 2004; 351:1607-18. [PubMed: 15483279]

14. Eggebrecht H, Pamler R, Zipfel B, et al. Thoracic aorta endografts: variations in practice among medical specialists. Catheter Cardiovasc Interv. 2006; 68:843-52. [PubMed: 17089408]

15. Dexter F, Dexter EU, Masursky D, Nussmeier NA. Systematic review of general thoracic surgery articles to identify predictors of operating room case durations. Anesth Analg. 2008; 106:1232-41. [PubMed: 18349199]

16. Zielinski M, Kuzdzal J, Szlubowski A, Soja J. Transcervical-subxiphoid-videothoracoscopic "maximal" thymectomy-operative technique and early results. Ann Thorac Surg. 2004; 78:404-9. discussion 409-10. [PubMed: 15276485]

17. Greenberg CC, Regenbogen SE, Studdert DM, et al. Patterns of communication breakdowns resulting in injury to surgical patients. J Am Coll Surg. 2007; 204:533-40. [PubMed: 17382211]

18. Emery SP, Kreutzer J, McCaffrey FM. The learning curve for a fetal cardiac intervention team. Minimally Invasive Surgery. 2010; 2010:1-5.

19. Mazzocco K, Petitti DB, Fong KT, et al. Surgical team behaviors and patient outcomes. Am J Surg. 2009; 197:678-85. [PubMed: 18789425] 
Table 1

\section{CPT Codes Used to Identify EVAR Patients}

\begin{tabular}{|c|c|}
\hline CPT Code & Operation \\
\hline 34800 & Endovascular repair of infrarenal abdominal aortic aneurysm or dissection; using aorto-aortic tube prosthesis \\
\hline 34802 & Endovascular repair of infrarenal abdominal aortic aneurysm or dissection; using modular bifurcated prosthesis ( 1 docking limb) \\
\hline 34803 & Endovascular repair of infrarenal abdominal aortic aneurysm or dissection; using modular bifurcated prosthesis ( 2 docking limbs) \\
\hline 34804 & Endovascular repair of infrarenal abdominal aortic aneurysm or dissection; using unibody bifurcated prosthesis \\
\hline 34805 & Endovascular repair of infrarenal abdominal aortic aneurysm or dissection; using aorto-uni-iliac or aorto-unifemoral prosthesis \\
\hline 34825 & $\begin{array}{l}\text { Placement of proximal or distal extension prosthesis for endovascular repair of infrarenal abdominal aortic or iliac aneurysm, } \\
\text { false aneurysm, or dissection; initial vessel }\end{array}$ \\
\hline 34826 & $\begin{array}{l}\text { Placement of proximal or distal extension prosthesis for endovascular repair of infrarenal abdominal aortic or iliac aneurysm, } \\
\text { false aneurysm, or dissection; each additional vessel (List separately in addition to code for primary procedure) }\end{array}$ \\
\hline 34830 & $\begin{array}{l}\text { Open repair of infrarenal aortic aneurysm or dissection, plus repair of associated arterial trauma, after unsuccessful endovascular } \\
\text { repair; tube prosthesis }\end{array}$ \\
\hline 34831 & $\begin{array}{l}\text { Open repair of infrarenal aortic aneurysm or dissection, plus repair of associated arterial trauma, after unsuccessful endovascular } \\
\text { repair; aorto-bi-iliac prosthesis }\end{array}$ \\
\hline 34832 & $\begin{array}{l}\text { Open repair of infrarenal aortic aneurysm or dissection, plus repair of associated arterial trauma, after unsuccessful endovascular } \\
\text { repair; aorto-bifemoral prosthesis }\end{array}$ \\
\hline 34833 & $\begin{array}{l}\text { Open iliac artery exposure with creation of conduit for delivery of aortic or iliac endovascular prosthesis, by abdominal or } \\
\text { retroperitoneal incision, unilateral }\end{array}$ \\
\hline 34834 & Open brachial artery exposure to assist in the deployment of aortic or iliac endovascular prosthesis by arm incision, unilateral \\
\hline 34900 & $\begin{array}{l}\text { Endovascular repair of iliac artery (e.g., aneurysm, pseudoaneurysm, arteriovenous malformation, trauma) using ilio-iliac tube } \\
\text { endoprosthesis }\end{array}$ \\
\hline
\end{tabular}


Table 2

Preoperative Risk Factors by Single Versus Multiple Specialist Participation in EVAR

\begin{tabular}{lccc}
\hline Characteristic & Single Specialty $(\mathbf{n}=\mathbf{7 , 0 8 6})$ & Multiple Specialties (n = 183) & P value \\
\hline Age (years) & $73.6 \pm 8.6$ & $73.9 \pm 9.4$ & 0.601 \\
Female & $17.6 \%$ & $20.8 \%$ & 0.263 \\
Emergent procedure & $6.3 \%$ & $7.1 \%$ & 0.657 \\
Dissection diagnosis & $0.5 \%$ & $0.6 \%$ & $0.602^{*}$ \\
Rupture diagnosis & $3.3 \%$ & $3.8 \%$ & 0.688 \\
Creatinine (mg/dL) & $1.25 \pm 0.79$ & $1.30 \pm 1.11$ & 0.554 \\
Preoperative dialysis & $1.5 \%$ & $0 \%$ & $0.064^{*}$ \\
Severe COPD & $18.5 \%$ & $14.8 \%$ & 0.201 \\
Current smoker & $28.6 \%$ & $28.4 \%$ & 0.958 \\
Congestive heart failure & $1.5 \%$ & $1.1 \%$ & $>0.999^{*}$ \\
White blood cell count $\left(1000 / \mathrm{cm}^{3}\right)$ & $7.7 \pm 2.9$ & $8.5 \pm 4.2$ & 0.014 \\
ASA 4 & $21.1 \%$ & $20.8 \%$ & 0.906 \\
ASA 5 & $1.2 \%$ & $0.6 \%$ & $0.727^{*}$ \\
Hypertension requiring oral medication & $78.5 \%$ & $80.9 \%$ & 0.436 \\
$>10 \%$ loss of body weight & $1.8 \%$ & $3.3 \%$ & $0.149^{*}$ \\
Sepsis & $0.6 \%$ & $1.6 \%$ & $0.114^{*}$ \\
Dependent functional status & $6.3 \%$ & $6.6 \%$ & 0.866 \\
\hline
\end{tabular}

Data are displayed as percent for categorical variables and mean \pm standard deviation for continuous variables.

$P$ values were obtained by $\chi^{2}$ or $t$ test depending on variable type.

*Fisher's exact test.

COPD, chronic obstructive pulmonary disease; ASA, American Society of Anesthesiologists physical status classification. 
Table 3

Intraoperative Characteristics by Single Versus Multiple Specialist Participation in EVAR

\begin{tabular}{lccc}
\hline Characteristic & Single Team $(\mathbf{n}=\mathbf{7 0 8 6})$ & Multiple Specialties $(\mathbf{n}=\mathbf{1 8 3})$ & $\boldsymbol{P}$ value \\
\hline Transfused intraoperatively & $15.5 \%$ & $20.2 \%$ & 0.084 \\
General surgeon ( $v s$ vascular) & $2.4 \%$ & $2.8 \%$ & 0.626 \\
Iliac or brachial artery exposure & $1.9 \%$ & $6.7 \%$ & $<0.001^{*}$ \\
Time in room (minutes) & $224.4 \pm 87.3$ & $238.2 \pm 91.2$ & 0.034 \\
Converted to open repair & $1.8 \%$ & $2.7 \%$ & $0.390^{*}$ \\
\hline
\end{tabular}

Data are displayed as percent for categorical variables and mean \pm standard deviation for continuous variables.

$P$ values were obtained by $\chi^{2}$ or $t$ test depending on variable type.

* Fisher's exact test. 
Table 4

Multivariate Model of 30-Day Mortality after EVAR

\begin{tabular}{lclc}
\hline Risk Factor & Odds Ratio & $\mathbf{9 5 \%}$ Confidence Interval & $\boldsymbol{P}$ value \\
\hline Multi-specialty operative team & 2.35 & $1.08-5.11$ & 0.031 \\
ASA class 4/5 & 2.80 & $2.06-3.82$ & $<0.001$ \\
White blood cell count $\left(1000 / \mathrm{cm}^{3}\right)$ & $1.16^{*}$ & $1.04-1.29$ & 0.008 \\
Emergent procedure status & 4.06 & $2.57-6.41$ & $<0.001$ \\
$>10 \%$ loss of body weight & 2.58 & $1.28-5.20$ & 0.008 \\
Female gender & 1.70 & $1.17-2.48$ & 0.006 \\
Dependent functional status & 2.48 & $1.63-3.76$ & $<0.001$ \\
Age (years) & $1.23 *$ & $1.93-7.25$ & 0.016 \\
Iliac or brachial artery exposure & 3.74 & $1.93-7.25$ & $<0.001$ \\
\hline
\end{tabular}

* Odds ratios are displayed per standard deviation change for continuous variables.

ASA, American Society of Anesthesiologists physical status classification. 